\title{
Tumor treating fields in neuro-oncology: integration of alternating electric fields therapy into promising treatment strategies
}

\author{
Tamar R. Berger, Eric T. Wong \\ Brain Tumor Center \& Neuro-Oncology Unit, Beth Israel Deaconess Medical Center, Harvard Medical School, Boston, MA, USA \\ Contributions: (I) Conception and design: ET Wong; (II) Administrative support: None; (III) Provision of study materials or patients: None; \\ (IV) Collection and assembly of data: None; (V) Data analysis and interpretation: None; (VI) Manuscript writing: Both authors; (VII) Final approval \\ of manuscript: Both authors. \\ Correspondence to: Eric T. Wong, MD. Brain Tumor Center \& Neuro-Oncology Unit, Beth Israel Deaconess Medical Center, 330 Brookline Avenue, \\ Harvard Medical School, Boston, MA 02215, USA. Email: ewong@bidmc.harvard.edu.
}

\begin{abstract}
As the most recent innovation in cancer therapy that utilizes properties of the electromagnetic spectrum, tumor treating fields (TTFields) are non-invasive alternating electrical fields that target rapidly dividing tumor cells. In the patient, TTFields are delivered as regional treatment via two pairs of orthogonally positioned transducer arrays applied to the head or elsewhere on the body surface. Side effects are primarily localized to the skin. Since the initial proof-of-concept study published in 2007 , the use of TTFields has become integrated into the standard-of-care multi-modality treatment of glioblastoma (GBM). In this review, we summarize the theory behind TTFields and describe how key mechanistic data helped guide pivotal clinical trials. We also highlight potential future applications.
\end{abstract}

Keywords: Electric fields; glioblastoma (GBM); mitosis; computer modeling; clinical trial

Submitted Dec 20, 2019. Accepted for publication Feb 03, 2020.

doi: $10.21037 /$ cco.2020.02.04

View this article at: http://dx.doi.org/10.21037/cco.2020.02.04

\section{Introduction}

Devices that exploit the biological effects of the electromagnetic spectrum have been in use for the treatment of cancer. They include linear accelerators that emit ionizing radiation and lasers that produce microwaves for the thermal ablation of tumors. The therapeutic index of these treatments is defined by the conformality of the delivery, as in stereotactic radiosurgery and laser-induced thermal therapy (LITT), where most of the applied energy is concentrated at the tumor while sparing adjacent normal tissues. Consistent with this concept, a third type of device that emits alternating electric fields at $200 \mathrm{kHz}$, known as tumor treating fields (TTFields), is the newest addition to this series of devices (Figure 1). It works by disrupting tumor cells as they undergo mitosis by interrupting proteins with large dipole moments that are critical for executing cytokinesis and segregation of sister chromatids. Past clinical trials have shown efficacy in prolonging survival of patients and TTFields have been approved by the United States FDA for the treatment of recurrent and newly diagnosed glioblastomas (GBMs) in 2011 and 2015, respectively. In addition, TTFields have been approved for mesothelioma in 2019 and they are being applied in clinical trials to other intracranial malignancies such as metastatic non-small cell lung cancer (NSCLC) as well as extracranial malignancies including advanced stage NSCLC, pancreatic carcinoma and ovarian cancer.

The goal of this review is to cover preclinical and clinical data accumulated thus far on TTFields. The preclinical data include the direct and indirect mechanisms of action. A discussion of the quantitative estimation of electric field distribution by finite element modeling is also included. Past clinical trial data will be critically evaluated, while emerging therapies and future directions will also be mentioned. 


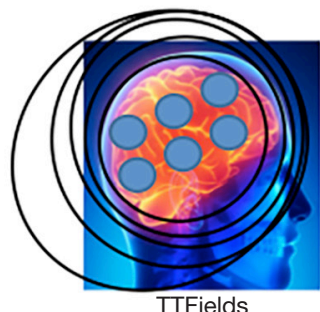

Biochemical Effect: membrane
depolarization Wavelength $(\mathrm{m}) \quad$ Antimitotic

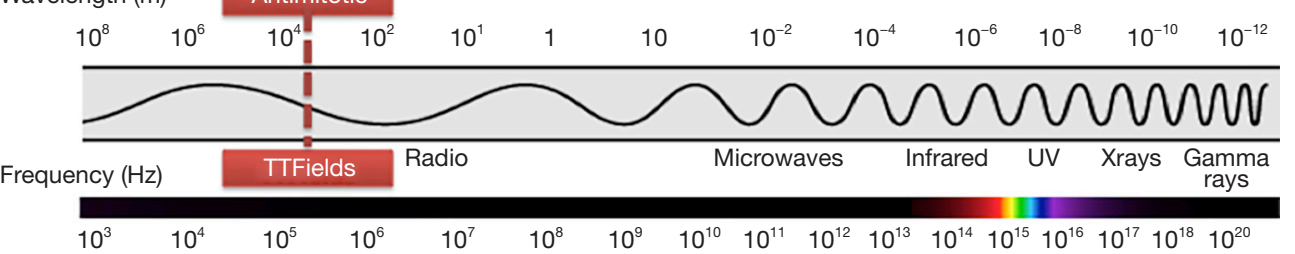

Figure 1 Brain tumor treatments using the electromagnetic spectrum. Conventional ionizing radiation has a frequency in the range of $\mathrm{EHz}$, or $10^{18}$ hertz, while LITT uses microwaves in the range of $\mathrm{GHz}$ (or $10^{9}$ hertz). TTFields use a much lower frequency at the $200 \mathrm{kHz}$ ( $\mathrm{kHz}$ or $10^{3}$ hertz) for GBM. TTFields, tumor treating fields; EHz, exahertz; LITT, laser-induced thermal therapy; GHz, gigahertz; kHz, kilohertz.

\section{Mechanisms of action}

The biological effect of TTFields was first observed by Dr. Yoram Palti from the Technion University in Israel. When the frequency of the alternating electric fields was tuned to $100-300 \mathrm{kHz}$, he observed dividing tumor cells underwent violent blebbing and eventual cell death (1). This led to further investigation of the direct and indirect cell biology effects of this poorly understood frequency band within the electromagnetic spectrum.

\section{Direct effects of TTFields on cancer cell fate}

TTFields directly disrupt the fundamental electromechanical properties that facilitate cell cycle division which leads to tumor growth. This effect causes cellular blebbing during mitosis. A series of experiments demonstrated that maximum blebbing can be induced by TTFields when the long axis of the dividing cell is aligned parallel to the direction of the fields $(1,2)$. Furthermore, the response is also dependent on cell size, with maximal growth inhibition at a frequency of $200 \mathrm{kHz}$ for cells of smaller size (i.e., F98 rat glioma as well as U87 and U118 human glioma cells) while it is at a slightly lower frequency of $100 \mathrm{kHz}$ for larger cells (i.e., B16F1 melanoma cells) (3). Initially, these cells were thought to undergo apoptotic blebbing.
However, they did not immediately die or dissociate from the tissue culture dish. On the contrary, they remained attached to the bottom of the dish. Immunofluorescent staining of these cells, using antibodies against microtubules and DAPI dye for DNA, demonstrated abnormal mitotic apparati. Multiple aberrant chromosome arrangements were observed, including polypoid prophase, rosettes, poorly-separated chromatids in metaphase, multi-spindled metaphase, single-spindled metaphase, asymmetric anaphase and disorganized microtubules (Figure 2). These changes in the architecture of mitotic spindles and chromosomes were thought to arise from disruption of tubulin complexes. This is because tubulin has a large dipole moment of 2,166 Debeyes and it is a building block of microtubules and mitotic spindles. Although treated cells did not undergo apoptosis immediately, they eventually died due to irreversible damage accumulated under prolonged exposure to TTFields.

Another important target of TTFields is septin, a large heterotrimeric protein assembled at the equatorial midline of the dividing tumor cell during cytokinesis, which occurs between anaphase and telophase of the $M$ cycle (4). Septin plays a key role in positioning the cytokinetic cleavage furrow and governing its contraction at the midline. It has a large dipole moment of 2,711 Debeyes and therefore makes 

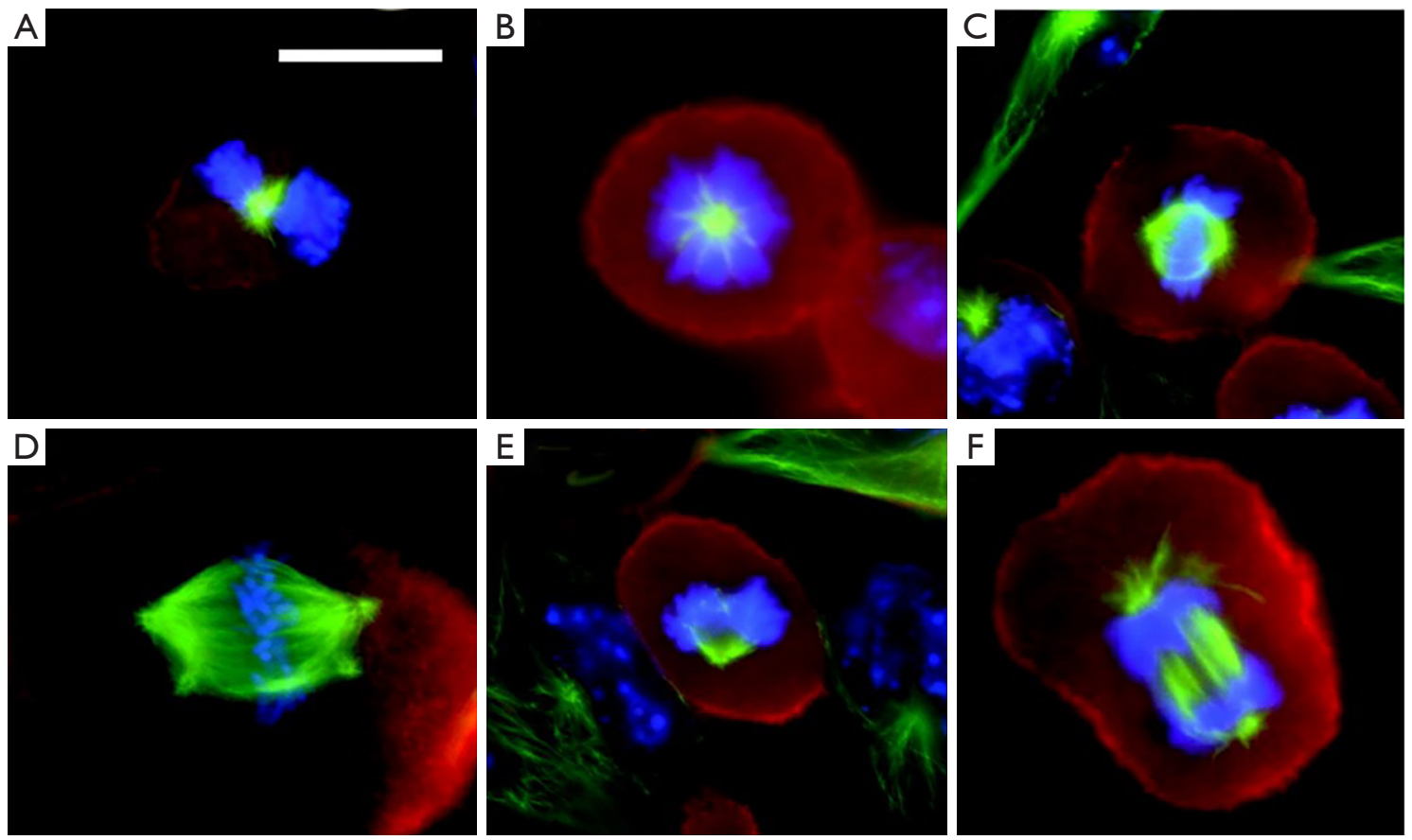

Figure 2 Immunohistochemical staining of aberrant mitotic figures as a result of TTFields. (A-F) Melanoma cells were stained with monoclonal antibodies for microtubules (green), actin (red), and DNA (blue) demonstrating polyploid prophase (A), rosette (B), poorly separated metaphase (C), multi-spindled metaphase (D), single-spindled metaphase (E) and asymmetric anaphase (F). Scale bar: $10 \mu m$. [Reprinted with permission from Reference (1)]. TTFields, tumor treating fields.

it susceptible to displacement by TTFields. Indeed, using fluorescent antibody under confocal microscopy, septin 7 localization at the midline of MDA-MB-231 and HeLa cells was reduced at a frequency of $150 \mathrm{kHz}$ but normal at $500 \mathrm{kHz}(2)$. The dispersal of septin results in disorganized and ectopic contractions throughout the plasma membrane, causing violent cytoplasmic blebbing seen in the dividing tumor cell during anaphase and telophase (Figure 3).

The micro-mechanical forces induced by TTFields can trigger a cytoplasmic stress response in the dividing tumor cell. This stress response is characterized by mitochondria with swollen matrices, decreased intracellular ATP levels, development of autophagosomes and expression of the endoplasmic reticulum stress marker GRP78 $(5,6)$. GRP78 is a major mediator of the unfolded protein response, which is activated by proteotoxic stress in the endoplasmic reticulum (7). In addition, TTFields applied to U87 and A172 glioma cells resulted in elevated levels of phosphorylated AMPK, a sensor of cellular bioenergetics that is activated by a variety of metabolic stresses and a master regulator of autophagy (6). Collectively, the data indicate that TTFields induce cytoplasmic stress in dividing tumor cells and cause stress-related adaptive responses that can be exploited therapeutically.

The application of TTFields also increases membrane permeability of tumor cells. Bioluminescence imaging revealed that TTFields treatment of U87 glioma cells increases their uptake of 5 -aminolevulinic acid or a fusion protein of GFP and firefly luciferase after 6 hours (8). Scanning electron microscopy also revealed an increase in the number and size of membrane pores (8). Interestingly, there is an upper limit to the size of the induced membrane pores and the largest ones only allow the diffusion of proteins with a maximum molecular weight of $40 \mathrm{kDa}(8)$. This process is also reversible within 24 hours after termination of TTFields (8).

Although TTFields primarily affect cytoplasmic proteins, they also influence the genome integrity of treated cells. This may be a result of the disruption of the regulatory proteins that shuttle back-and-forth between the cytoplasm and the nucleus. Indeed, in a series of NSCLC cell lines, TTFields treatment resulted in diminished repair of DNA doublestrand breaks as a result of downregulated BRCA1 signaling, with some of the cell lines (i.e., H157 and H4006) more 
sensitive than others (i.e., A549, H1650 and H1299) (9). Gene expression analysis revealed reduced expression of important regulators of mitosis and genes for replication stress under TTFields (10). This causes delayed DNA damage repair when both TTFields and ionizing radiation were applied to U118 and LN18 glioma cell lines (11).

\section{Indirect effects of TTFields and their therapeutic potential}

The direct effects of TTFields may condition the tumor cells to make them more susceptible for treatment when combined with other types of anti-cancer modalities. First, TTFields may improve outcomes for bulk surgical resection through enhanced uptake of fluorescent ligands that delineate tumor boundaries. Specifically, 5 -aminolevolenic acid, an FDA-approved ligand for fluorescence-guided surgical resection, had enhanced permeability in human GBM cell lines treated with TTFields compared to non-treated cell lines (8). Normal human cells did not demonstrate a TTFields-specific increase in 5-aminolevolenic acid uptake, suggesting that the application of pre-operative TTFields may help to improve the surgical excision of GBM $(12,13)$.

Another important TTFields pre-conditioning of tumor cells is associated with the diminished repair of DNA double-strand breaks $(9,11)$. Since an important effect of ionizing radiation is the induction of double-strand breaks, the use of TTFields during or shortly after completion of radiation in the patient may potentially augment the antitumor effect of radiation. Indeed, preclinical experiments have shown a synergistic anti-glioma effect when TTFields were applied after radiation and this is a result of delayed repair of radiation-induced DNA damage (11). Similarly, the combination of TTFields with traditional chemotherapeutic agents, such as paclitaxel, doxorubicin, cyclophosphamide, pemetrexed, cisplatin and bleomycin, has been shown to augment cytotoxicity in at least an additive fashion $(11,14,15)$.

Activation of the immune system is another important effect of TTFields. A number of preclinical experiments have demonstrated the induction of immunogenic cell death of tumor cells by TTFields and this effect is augmented when combined with anti-PD1 therapy $(16,17)$. This synergy is mediated by the expression of calreticulin on the surface of tumor cells and the secretion of alarmin HMGB1 into the tumor microenvironment (17-19). Supporting the notion of immune activation, the implantation of VX-2 tumors in the renal capsule of rabbits and the application of TTFields to the kidneys decreased the number of lung metastasis in these animals (14), making this phenomenon similar to the known abscopal effect from ionizing radiation (20). In addition, the use of higher doses of immunosuppressant dexamethasone in patients can potentially negate the benefit of TTFields (21).

Understanding the direct and indirect effects of TTFields on cancer cells and the interactions within the tumor microenvironment will help the design of future combination therapies. Current methodologies to study the mechanistic effects of TTFields include the Inovitro apparatus for applying alternating electric fields in customized tissue culture dishes as well as the Inovivo device for such application in rodent tumor models. Collectively, the data so far from both in vitro and in vivo experiments point to synergism with traditional anti-cancer therapies.

\section{Electric field modeling}

The intensity of alternating electric fields delivered in cell culture experimentation correlates with the number of disrupted tumor cells in mitosis (1). The in vitro distribution of the fields is uneven and the greatest disruption was observed when the fields are parallel to the long axis of the dividing cells; there is minimum disruption when applied perpendicular to the cells. The set up in the Inovitro apparatus consists of two pairs of opposing parallel plates oriented orthogonal to each other (22). Therefore, the number of disrupted cells is maximum at the center of the dish and minimum at each of the 4 corners. This arrangement mimics the transducer arrays placement in patients (23). Although the arrays have a fixed delivery of TTFields at $1 \mathrm{~V} / \mathrm{m}$ and this intensity cannot be adjusted, the fields can be either higher or lower than this value once they penetrate into the intracranial compartment. This is due to the "wave-like" property of alternating electric fields and the presence of two pairs of transducer arrays on the patient's scalp. When two opposing waves converge, they can summate when they are in phase or cancel out when they are out of phase. In addition, the intensity of the fields also depends on a number of factors, including (I) the local tissue properties, with conductivity (the ability to pass charges) more influential than permittivity (the ability to hold charges), (II) the geometry of the interface between tissues with different conductivity values, and (III) proximity to the transducers on the scalp. Because of these complex interactions, the quantification of TTFields at the gross tumor volume (GTV) cannot be solved analytically and can 


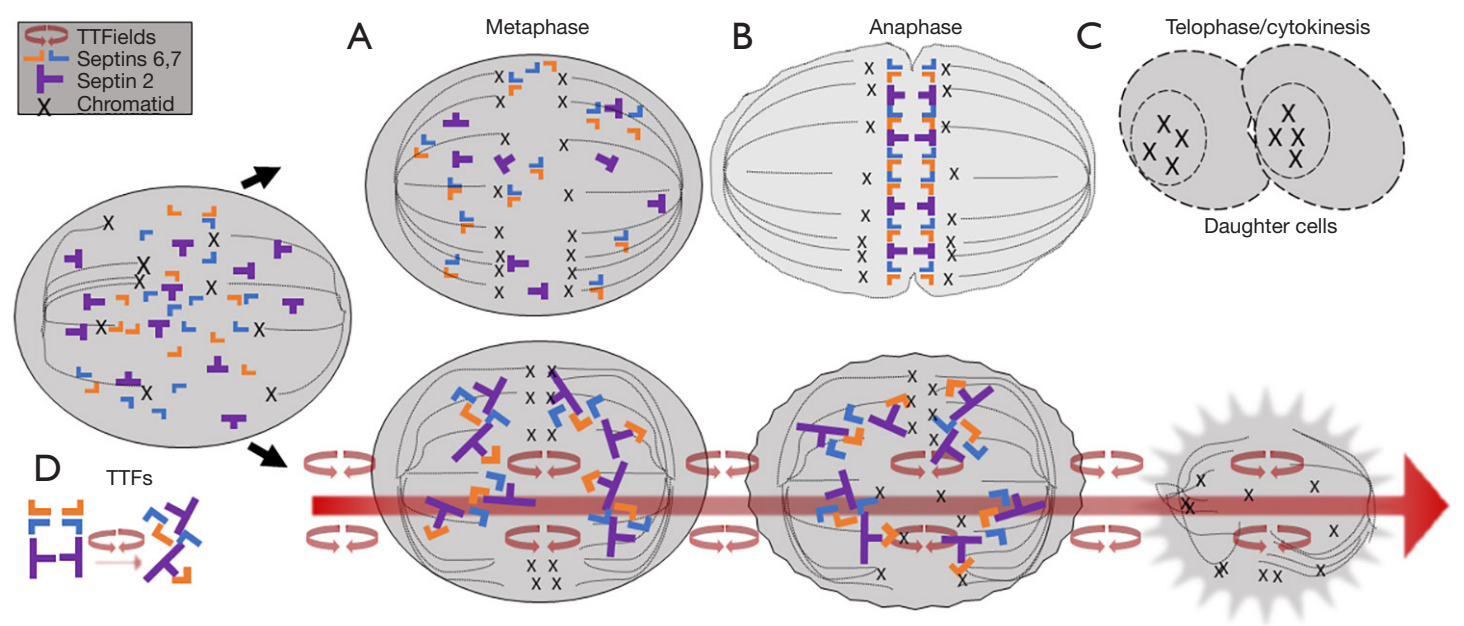

Figure 3 TTFields disrupt septin localization leading to mitotic exit and eventual cell death. In anaphase, septin 2, 6, 7 complexes are recruited to the spindle midline and form a fibrous lattice through lateral interactions between parallel septin filaments (top panel: A, B); this facilitates contractile elements within the cytokinetic furrow to facilitate progression to telophase/cytokinesis (top panel: C); TTFields induce rotational movement within the parallel fibers at a slightly more than a right angle to their lateral axis. As such, the alternating electric fields (arrows) blocks lattice formation by disrupting the binding of individual fibers to each other (bottom panel: A, B, D); in the absence of proper septin function, contractile elements of the cytokinetic furrow are dispersed from the equatorial midline of the cell, resulting in ectopic furrow malfunction that leads to violent membrane contractions at the onset of anaphase followed by aberrant mitotic exit (bottom panel: C). TTFields, tumor treating fields.

only be determined by computational approximation.

Finite element analysis is a method often used to approximate TTFields in the intracranial space. There are two approaches that are available for this analysis: (I) segmentation of the intracranial structures of each individual patient (23) or (II) segmentation of only the GTV that is subsequently incorporated into an idealized brain model (24). The first approach requires the importation of DICOM images of post-acquisition MRI MP-RAGE, $\mathrm{T} 1$ and $\mathrm{T} 2$ sequences into a segmentation software that allows the delineation of various brain structures along with the GTV and the necrotic core within the GTV. Upon completion of segmentation, a three-dimensional finite-element mesh is generated and the meshed brain model is then exported to a Multiphysics solver for finite element analysis (Figure 4). The second approach is similar to the first except for the assumption that the variability of the intracranial structures is minimal, and the scalp and calvarium have similar thicknesses between patients. Therefore, only the GTV is contoured and segmented, and a three-dimensional mesh is generated from the segmented GTV that is incorporated into a common brain background. The key difference between the two approaches is that the first takes into account patient heterogeneity of intracranial neuroanatomical structures but the process of contouring various structures is time-consuming. In contrast, the second approach takes less time to achieve a three-dimensional mesh because the contoured idealized brain can be applied to multiple patients-only the GTVs are different among individual patients-and therefore this process saves time. There is yet no comparative study to determine which approach provides a more accurate measure of TTFields at the GTV or at various intracranial structures.

Plan Quality Metrics such as the electric field-volume histogram (EVH) can be used to quantitatively compare TTFields coverage and intensity between models derived from the same individual or from different patients. These metrics include area under the curve of the GTV or a particular structure in the $\mathrm{EVH}\left(\mathrm{E}_{\mathrm{AUC}}\right)$, the percentage volume receiving at least $150 \mathrm{~V} / \mathrm{m}\left(\mathrm{V}_{\mathrm{E} 150}\right)$ and the magnitude of electric field strength encompassing $95 \%\left(\mathrm{E}_{95 \%}\right), 50 \%$ $\left(\mathrm{E}_{50 \%}\right)$ or $20 \%\left(\mathrm{E}_{20 \%}\right)$ of the GTV or a particular structure's volume (Figure 4) (25). Other metrics that can be used for quantitative comparisons include the specific absorption ratio-volume histogram and the current density-volume histogram $(25,26)$. Collectively, these parameters can provide valuable insights on the effect of TTFields at the GBM or other intracranial sites. 

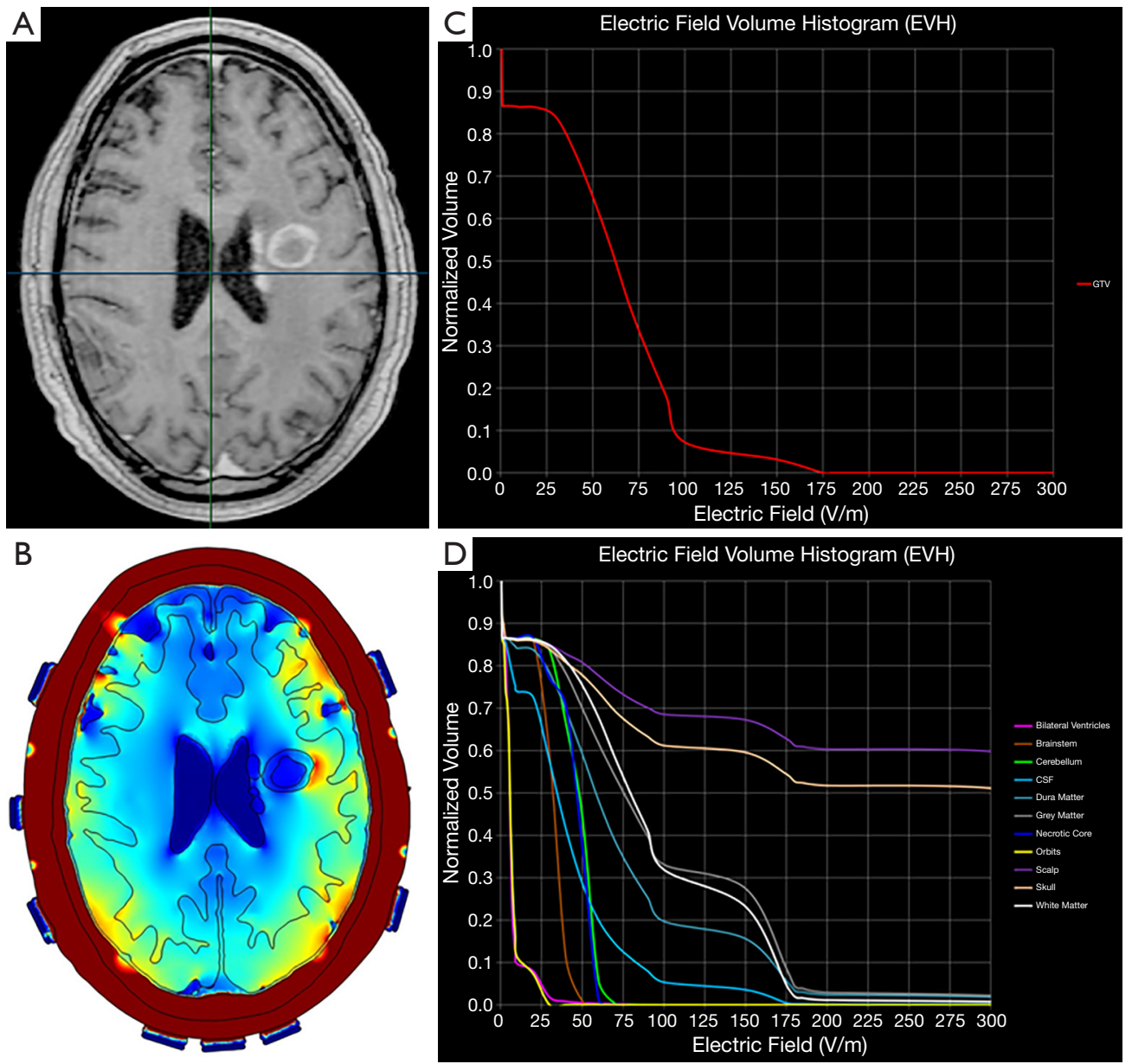

Figure 4 TTFields treatment planning and Plan Quality Metrics. The post-gadolinium T1-weighted image was used for autosegmentation and contouring of the GTV and other intracranial structures (A); a heatmap is generated delineating the electric field intensity within the brain (B); the EVH reveals the volume-dose relationship in the GTV (C) and other intracranial structures (D). TTFields, tumor treating fields; GTV, gross tumor volume; EVH, electric field-volume histogram.

\section{Results of past and present clinical trials}

The first-in-human study to demonstrate safety and feasibility of TTFields was conducted in 10 patients with recurrent GBM (3). Treatment-related adverse events consisted of mild to moderate dermatitis on the scalp in 9 of 10 patients. The entire cohort had a median progression-free survival (PFS) of 26.1 weeks, median overall survival (OS) of 62.2 weeks and 1-year survival rate of $68 \%$, which was promising when compared to the benchmark PFS of 10 weeks, OS of 30 weeks and 1 -year survival rate of $21 \%$ (27). The favorable results from this pilot study led to the implementation of the EF-11 randomized phase III clinical trial for recurrent GBM patients (NCT00379470) (28). The EF-11 clinical trial included 237 patients with a median age of 54 and Karnofsky Performance Score (KPS) of 70. Primary endpoint was OS with secondary endpoints evaluating PFS at 6 months, 1-year survival, radiologic response rate and quality of life. Comparisons were made to a similar baseline control group receiving physician's choice chemotherapy. The results showed that TTFields monotherapy was non-inferior to physician's choice chemotherapy, and with improved treatment tolerability and significantly fewer adverse events. There was a trend towards improved OS, 6.6 vs. 6.0 months, but it was not statistically significant (28). Nevertheless, the trial data was presented to the United States FDA in a panel meeting convened in 2011 and received a positive review based 
A

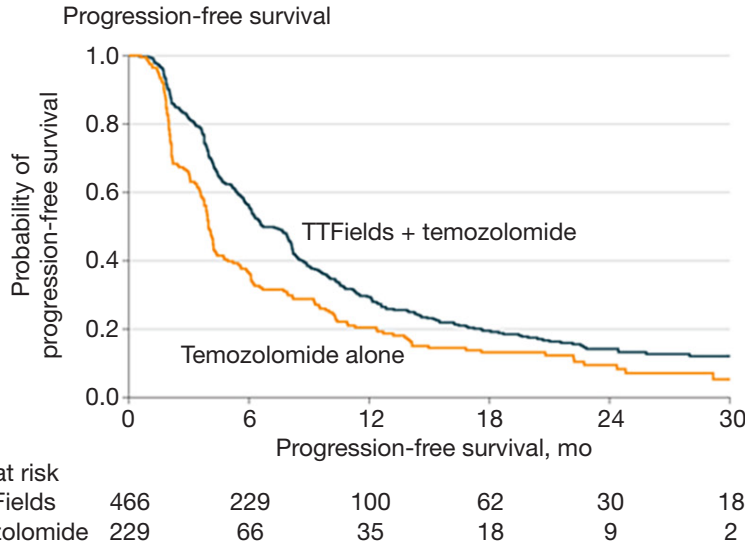

B

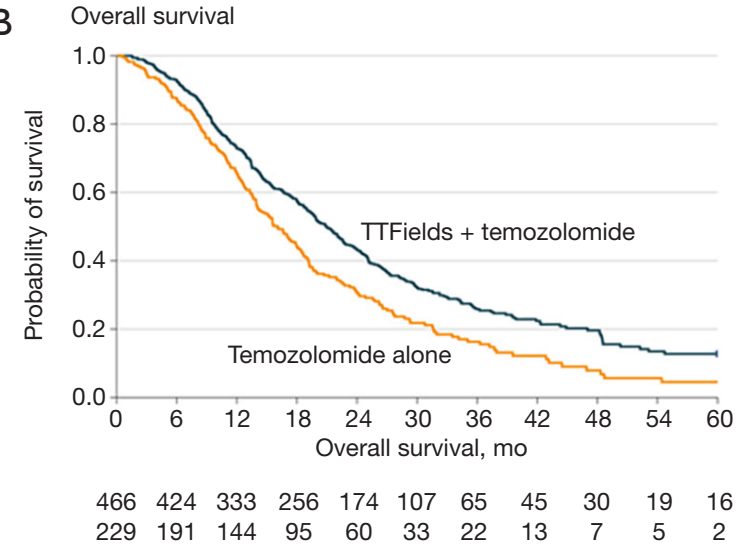

Figure 5 Kaplan-Meier survival curves showing a survival benefit for patients in EF-14. (A) Median PFS from randomization for the TTFields plus temozolomide group was 6.7 months and was 4.0 months for the temozolomide alone group (HR: 0.63; 95\% CI: 0.52-0.76; $\mathrm{P}<0.001$ ); (B) median OS from randomization was 20.9 for the TTFields plus temozolomide group vs. 16.0 months for the temozolomide alone group (HR: 0.63; 95\% CI: 0.53-0.76; P<0.001). Median follow up was 44 (range, 25-91) months in both groups. (Reprinted with permission from: Stupp R, Taillibert S, Kanner A, et al. Effect of tumor-treating fields plus maintenance temozolomide vs maintenance temozolomide alone on survival in patients with glioblastoma: a randomized clinical trial. JAMA 2017;318:2306-16.). TTFields, tumor treating fields; HR, hazard ratio; PFS, progression-free survival; OS, overall survival.

on the better toxicity profile compared to chemotherapy. TTFields treatment was therefore first approved in 2011 for use in patients with recurrent GBM.

Shortly after the initial FDA approval of the Optune ${ }^{\mathrm{TM}}$ device that delivers TTFields, a patient registry was established to capture treatment outcome. The Patient Registry Dataset (PRiDe) collected data on 457 patients who received TTFields for their recurrent GBM. Interestingly, PRiDe patients had a longer median OS than those in the TTFields monotherapy arm in EF-11, 9.6 vs. 6.6 months respectively (29). Even though the two populations are not statistically comparable because they received treatments under different conditions and at asynchronous time points, it is still remarkable that the PRiDe patients appeared to live much longer and there are a number of explanations for that. First, compared to EF-11, a higher proportion of patients in PRiDe received TTFields at first recurrence, $9 \%$ vs. $33 \%$, rather than at third to fifth recurrence, $43 \%$ vs. 27\%, respectively (29). Second, patients in PRiDe most likely received other concurrent treatments, including stereotactic radiosurgery, chemotherapies, bevacizumab or any of the combination, but these data were not captured in the dataset. Despite its limitation, PRiDe offers a glimpse of patient outcome as TTFields were used in real-world clinical settings. It confirmed the positive prognostic factors previously identified in the post hoc analysis of EF-11, which were treatment compliance of $\geq 75 \%$, first or second recurrence and higher KPS; the only negative prognostic factor was prior bevacizumab use (30).

The next randomized clinical trial, EF-14 (NCT00916409), expanded the subject population to patients with newly diagnosed GBM, comparing treatment with TTFields plus adjuvant temozolomide versus temozolomide alone (31). This trial was the largest in neuro-oncology at the time and included a total of 695 patients who had already completed initial radiation therapy with concomitant temozolomide. The study arms had 466 patients in the TTFields plus adjuvant temozolomide group versus 229 patients in the temozolomide alone group in a 2:1 ratio. The primary endpoint was PFS, with results demonstrating an increase in the PFS of 3.1 months in the population treated with TTFields and adjuvant temozolomide compared to those who received temozolomide alone [7.1 vs. 4.0 months; hazard ratio (HR): 0.62; $\mathrm{P}=0.001]$. Secondary endpoints included OS (20.5 vs. 15.6 months; HR: 0.64, $\mathrm{P}=0.004)$ and 2-year survival (43\%, $95 \%$ CI: $36-50 \%$ vs. $29 \%$, $95 \%$ CI: 21-38\%) (31) (Figure 5). Quality of life was maintained in the TTFields arm without documentation of significant toxicity or adverse events. Based on these data, the FDA approved in 2015 TTFields in combination with temozolomide for newly diagnosed GBM following initial surgery and radiation therapy with concomitant daily temozolomide (31). 
The EF-14 trial also included a robust health-related quality of life assessment using the European Organization for Research and Treatment of Cancer (EORTC) QLQ-C30 questionnaires (32). No significant difference was seen between the two cohorts except for scalp irritation in the TTFields-treated subjects (33). Measures for global health status, physical functioning, emotional functioning, pain and leg weakness all favored the TTFields-treated group, while role and social functioning were not affected by TTFields $(33,34)$. Therefore, when added in the adjuvant setting, TTFields did not have a negative influence on patients' health-related quality of life except for scalp pruritis.

A number of post hoc analyses were performed from the EF-14 dataset. First, TTFields compliance was again an important prognostic factor. There was a linear relationship between percent compliance and OS, starting with a median OS of 18.0 months at $50-60 \%$ compliance to 24.9 months at $>90 \%$ compliance; those achieving a compliance of $>90 \%$ had a 5 -year survival rate of almost $30 \%$ (35). Second, dosimetry analysis showed a correlation between TTFields power density and survival. Power density is calculated as the product of TTFields intensity, tissue-specific conductivity and treatment compliance. The OS and PFS were significantly prolonged when local minimum power density at the GTV was $\geq 0.77 \mathrm{~mW} / \mathrm{cm}^{3}$ (36). However, only $340(73 \%)$ of the entire TTFields-treated population $(n=466)$ had MRI qualities acceptable for analysis and the inclusion of the missing data could potentially alter the significance of this correlation. Furthermore, the outcome of this analysis remains to be confirmed in a validation cohort.

There is immense enthusiasm in combining TTFields with currently available therapies for recurrent GBM and a number of studies have documented the use in this fashion (Table 1). An objective response with a $50 \%$ decrease in tumor size was observed in a patient treated with TTFields in combination with 6-thioguanine, lomustine, capecitabine and celecoxib (37). A retrospective review was also conducted in 48 patients with recurrent GBM who received TTFields in conjunction with either a triple-drug regimen of bevacizumab, irinotecan and temozolomide or bevacizumabbased regimens consisting of bevacizumab with or without irinotecan or lomustine. Patients treated with TTFields and triple-drug regimen had an improved survival compared to those treated with any of the bevacizumab-based regimens, with a median PFS of $6.6 v s .5 .1$ months and a median OS of 32.5 vs. 17.8 months, respectively (38). At the very least, these studies showed that combining TTFields and other conventional modalities of treatment is safe and without unexpected toxicities. Therefore, TTFields combination therapies are now being addressed in several prospective studies, with a specific focus on TTFields in combination with bevacizumab for recurrent GBM (NCT02663271, NCT01894061 and NCT01925573) as well as TTFields with stereotactic radiosurgery (NCT01925573). For patients with newly diagnosed unresectable GBM, there is a study evaluating the combination of TTFields with bevacizumab and temozolomide (NCT02343549). In addition, there are several clinical trials that are currently studying an adjunct role for immune-modulatory therapy and TTFields in GBM. One trial is studying the effect of personalized mutation-derived antigen vaccine in combination with TTFields after radiation and chemotherapy (NCT03223103). Another trial is currently recruiting patients with recurrent GBM to study the role of TTFields in combination with nivolumab with or without ipilimumab (NCT03430791). For patients with newly diagnosed GBM, there is a trial investigating the effect of combining pembrolizumab with TTFields and adjuvant temozolomide (NCT03405792).

A number of non-therapeutic trials are aimed to identify imaging and pathology biomarkers in the GBM population treated with TTFields. For example, a study using highresolution MRI seeks to identify imaging parameters as potential predictors of response to TTFields (NCT02441322). Gene expression changes and pathological changes are also being studied in patients with newly diagnosed or recurrent GBM who were treated with TTFields (NCT01954576 and NCT01954576).

Clinical trials using TTFields are also being conducted for the treatment of malignancies other than GBM (Table 1), including brain metastases from NSCLC or METIS (NCT02831959), stage IV systemic NSCLC following platinum failure or LUNAR (NCT02973789), locally advanced pancreatic carcinoma with gemcitabine-based treatment or PANOVA (NCT01971281), and platinumresistant ovarian cancer with weekly paclitaxel or INNOVATE (NCT03940196). In addition, a single-arm phase II study of TTFields with pemetrexed and platinum agent for malignant pleural mesothelioma has been completed (NCT02397928) and the United States FDA has approved the use of NovoTTF ${ }^{\mathrm{TM}}$-100L system in this population in 2019 (39).

\section{Emerging therapies and future directions}

Based on the known mechanisms of action of TTFields, 
Table 1 Summary of clinical trials incorporating TTFields with combination therapies for GBM and other malignancies

\begin{tabular}{|c|c|c|c|c|}
\hline Trial & Phase & Combination therapy & Disease & Primary outcome \\
\hline NCT02663271 & $\begin{array}{l}\text { Phase II (multi-center, } \\
\text { single arm) }\end{array}$ & Bevacizumab (pulsed) & $\begin{array}{l}\text { Bevacizumab-refractory } \\
\text { GBM }\end{array}$ & OS \\
\hline NCT01894061 & Phase II & Bevacizumab & $\begin{array}{l}\text { Recurrent GBM } \\
\text { (bevacizumab-naive) }\end{array}$ & PFS \\
\hline NCT02343549 & Phase II & Bevacizumab + temozolomide & $\begin{array}{l}\text { Newly diagnosed, } \\
\text { unresectable GBM }\end{array}$ & $\begin{array}{l}\text { Survival at } 12 \\
\text { months }\end{array}$ \\
\hline NCT03223103 & Phase la/lb & Mutation-derived antigen vaccine & Newly diagnosed GBM & $\begin{array}{l}\text { Dose-limiting } \\
\text { toxicities }\end{array}$ \\
\hline NCT03405792 & Phase II & $\begin{array}{l}\text { Pembrolizumab with adjuvant } \\
\text { temozolomide }\end{array}$ & Newly diagnosed GBM & PFS \\
\hline \multicolumn{5}{|c|}{ TTFields in other malignancies } \\
\hline $\begin{array}{l}\text { NCT02831959 } \\
\text { (METIS) }\end{array}$ & Phase III & Following stereotactic radiosurgery & $\begin{array}{l}\text { Brain metastases from } \\
\text { NSCLC }\end{array}$ & $\begin{array}{l}\text { Time to intracranial } \\
\text { progression }\end{array}$ \\
\hline $\begin{array}{l}\text { NCT02973789 } \\
\text { (LUNAR) }\end{array}$ & Phase III & $\begin{array}{l}\text { Standard therapies for stage IV } \\
\text { NSLCL }\end{array}$ & $\begin{array}{l}\text { Stage IV systemic NSCLC } \\
\text { following platinum failure }\end{array}$ & os \\
\hline $\begin{array}{l}\text { NCT01971281 } \\
\text { (PANOVA) }\end{array}$ & Phase II & Gemcitabine-based treatment & $\begin{array}{l}\text { Locally-advanced } \\
\text { pancreatic carcinoma }\end{array}$ & $\begin{array}{l}\text { Adverse events, } \\
\text { feasibility }\end{array}$ \\
\hline
\end{tabular}

TTFields, tumor treating fields; GBM, glioblastoma; PFS, progression-free survival; OS, overall survival; NSCLC, non-small cell lung cancer.

it is possible to rationally combine this therapy with other modalities of anti-cancer treatment. First, the direct antimitotic effect can potentially be augmented by the addition of other cytotoxic chemotherapies. For example, the combination of TTFields plus paclitaxel, doxorubicin or cyclophosphamide exerted an additive anti-mitotic effect on cell culture experiments $(14,15)$. This additive effect observed in the preclinical setting translated into a survival benefit among newly diagnosed GBM patients in the registration randomized phase III study comparing adjuvant TTFields plus temozolomide versus temozolomide alone (31). The additional clinical benefit of TTFields remains to be determined in the ongoing PANOVA study when TTFields is added to paclitaxel and gemcitabine for locally advanced pancreatic adenocarcinoma (NCT01971281). Radiation is another relevant combination that may directly result in an additive or synergistic anti-cancer effect. Radiation causes damage in DNA and generation of oxygen radicals, both of which can potentially augment the anti-mitotic effect of TTFields. Specifically, preclinical investigation has shown that TTFields delays radiation-induced DNA damage repair in GBM cells (11). Two phase I studies explored the feasibility of concurrent TTFields during the upfront radiation and daily temozolomide treatment of newly diagnosed GBM patients $(40,41)$. Therefore, fractionated radiotherapy and TTFields could potentially act synergistically to prolong survival in the GBM population. Furthermore, singlefraction or hypofractionated stereotactic radiosurgery delivers a large dose to metastases in a conformal fashion. Therefore, stereotactic radiosurgery and TTFields could 
also act synergistically in the population with brain metastasis from systemic malignancies.

Second, another strategy for treatment combination with TTFields utilizes the indirect effect of immunogenic cell death. The stress imposed onto tumor cells during mitosis results in the secretion of HMGB1, a DNA-damage response signal, into the tumor microenvironment as well as the expression of calreticulin onto the surfaces of tumor cells (18). Together, both processes allow the tumor cells to be recognized by the immune system, assuming that the patient still has intact anti-tumor immunity. HMGB1 recruits tumorassociated macrophages and biases them towards the M1 phenotype, inducing a proinflammatory anti-tumor response by the secretion of cytokines and activation of cytotoxic lymphocytes. Therefore, the combination of TTFields and checkpoint inhibitor may offer a superior anti-GBM effect than TTFields alone. By removing the inhibitory effect on anti-tumor immunity, checkpoint inhibitors enable $\mathrm{T}$ effector cells to exert a more robust anti-tumor effect.

The pro-inflammatory anti-tumor response to TTFields can be attenuated by large doses of dexamethasone. Indeed, dexamethasone at doses higher than $4 \mathrm{mg}$ is associated with the decreased survival in GBM patients (37). Other known immunosuppressants, such as rapamycin and everolimus, are also contraindicated in this population (42). Therefore, meticulous attention to limit the use of dexamethasone, as well as other immunosuppressants, is prudent in the management of the GBM population. In addition, drugs that can thwart the actions of cells that carry out immunosuppressive functions within the tumor microenvironment are probably beneficial in maximizing treatment efficacy. For example, COX-2 inhibition can block the local synthesis of prostanoids that enable the survival of regulatory $\mathrm{T}$ cells (Tregs) and myeloid derived suppressive cells (MDSCs), both of which exert their immunosuppressive functions either directly or indirectly within the local tumor microenvironment (43). Furthermore, 5-fluroururacil (5-FU) is a cytotoxic agent that can readily cross the blood brain barrier and is toxic to MDSCs. But the concentration of this drug within the local tumor microenvironment may not be achievable by routine intravenous or oral administration. Therefore, a gene therapy approach involving the injecting a replication defective retrovirus vocimagene amiretrorepvec that delivers an enzyme adenosine deaminase and taking an oral formulation of 5 -flurocytosine $(5-\mathrm{FC})$ that can be readily converted to 5 -FU by the enzyme may result in a higher local level of 5-FU (44). However, a phase III randomized study of vocimagene amiretrorepvec in recurrent high grade gliomas did not result in a survival advantage when compared to physician's choice salvage chemotherapies (45). Nevertheless, the addition of specific COX-2 celecoxib or local gene therapy approach that results in a higher local levels of 5-FU may potentiate the efficacy of TTFields.

\section{Conclusions}

TTFields have evolved from an observed laboratory phenomenon to bona fide treatment for GBM and other malignancies. The anti-cancer effect of TTFields is based on disruption of tumor cell mitosis at anaphase and telophase, triggering a cascade of downstream direct or indirect effects that interfere with tumor growth and activate antitumor immunity within the patient. The clinical efficacy of TTFields was first demonstrated in the treatment of GBM when applied together with adjuvant temozolomide in the newly diagnosed population. An additional indication for the treatment of malignant pleural mesothelioma was also approved recently. Furthermore, randomized clinical trials are being conducted for brain metastasis and other systemic malignancies. Therefore, TTFields will likely become one of the pillars of anti-cancer therapies alongside surgery, radiation, chemotherapy and immunotherapy.

\section{Acknowledgments}

Funding: This work is supported in part by $A$ Reason To Ride research fund and Musella Foundation.

\section{Footnote}

Provenance and Peer Review: This article was commissioned by the Guest Editor (Rimas V. Lukas) for the series "The Evolving Landscape of the Management of Glioblastoma" published in Chinese Clinical Oncology. The article has undergone external peer review.

Conflicts of Interest: Both authors have completed the ICMJE uniform disclosure form (available at http://dx.doi. org/10.21037/cco.2020.02.04). ETW reports grants from Novocure, Ltd., outside the submitted work. The other author has no conflicts of interest to declare.

Ethical Statement: The authors are accountable for all aspects of the work in ensuring that questions related to the accuracy or integrity of any part of the work are 
appropriately investigated and resolved.

Open Access Statement: This is an Open Access article distributed in accordance with the Creative Commons Attribution-NonCommercial-NoDerivs 4.0 International License (CC BY-NC-ND 4.0), which permits the noncommercial replication and distribution of the article with the strict proviso that no changes or edits are made and the original work is properly cited (including links to both the formal publication through the relevant DOI and the license). See: https://creativecommons.org/licenses/by-nc-nd/4.0/.

\section{References}

1. Kirson ED, Gurvich Z, Schneiderman R, et al. Disruption of cancer cell replication by alternating electric fields. Cancer Res 2004;64:3288-95.

2. Gera N, Yang A, Holtzman TS, et al. Tumor treating fields perturb the localization of septins and cause aberrant mitotic exit. PloS One 2015;10:e0125269.

3. Kirson ED, Dbalý V, Tovarys F, et al. Alternating electric fields arrest cell proliferation in animal tumor models and human brain tumors. Proc Natl Acad Sci U S A 2007;104:10152-7.

4. Mostowy S, Cossart P. Septins: the fourth component of the cytoskeleton. Nat Rev Mol Cell Biol 2012;13:183-94.

5. Silginer M, Weller M, Stupp R, et al. Biological activity of tumor-treating fields in preclinical glioma models. Cell Death Dis 2017;8:e2753.

6. Shteingauz A, Porat $Y$, Voloshin T, et al. AMPKdependent autophagy upregulation serves as a survival mechanism in response to tumor treating fields (TTFields). Cell Death Dis 2018;9:1074.

7. Casas C. GRP78 at the centre of the stage in cancer and neuroprotection. Front Neurosci 2017;11:177.

8. Chang E, Patel CB, Pohling C, et al. Tumor treating fields increases membrane permeability in glioblastoma cells. Cell Death Discov 2018;4:113.

9. Karanam NK, Srinivasan K, Ding L, et al. Tumor-treating fields elicit a conditional vulnerability to ionizing radiation via the downregulation of BRCA1 signaling and reduced DNA double-strand break repair capacity in non-small cell lung cancer cell lines. Cell Death Dis 2017;8:e2711.

10. Karanam NK, Ding L, Aroumougame A, et al. Tumor treating fields cause replication stress and interfere with DNA replication fork maintenance: Implications for cancer therapy. Transl Res 2020;217:33-46.

11. Giladi M, Munster M, Schneiderman RS, et al. Tumor treating fields (TTFields) delay DNA damage repair following radiation treatment of glioma cells. Radiat Oncol 2017;12:206.

12. Krieg RC, Uihlein D, Murthum T, et al. Improving the use of 5 -aminolevulinic acid (ALA)-induced protoporphyrin IX (PPIX) for the gastrointestinal tract by esterification--an in vitro study. Cell Mol Biol (Noisy-le-grand) 2002;48:917-23.

13. Stummer W, Pichlmeier U, Meinel T, et al. Fluorescenceguided surgery with 5-aminolevulinic acid for resection of malignant glioma: a randomised controlled multicentre phase III trial. Lancet Oncol 2006;7:392-401.

14. Kirson ED, Schneiderman RS, Dbalý V, et al. Chemotherapeutic treatment efficacy and sensitivity are increased by adjuvant alternating electric fields (TTFields). BMC Med Phys 2009;9:1.

15. Giladi M, Weinberg U, Schneiderman RS, et al. Alternating electric fields (tumor-treating fields therapy) can improve chemotherapy treatment efficacy in non-small cell lung cancer both in vitro and in vivo. Semin Oncol 2014;41 Suppl 6:S35-41.

16. Voloshin T, Yitzhaki OT, Kaynan N, et al. IMMU-59. Alternating electric fields (TTFields) induce immunogenic cell death resulting in enhanced antitumor efficacy when combined with anti-PD-1 therapy. Neuro Oncol 2017;19:vi126.

17. Giladi M, Voloshin T, Shteingauz A, et al. Alternating electric fields (TTFields) induce immunogenic cell death resulting in enhanced antitumor efficacy when combined with anti-PD-1 therapy. J Immunol 2016;196:75.26.

18. Swanson KD, Lok E, Wong ET. An overview of alternating electric fields therapy (novoTTF therapy) for the treatment of malignant glioma. Curr Neurol Neurosci Rep 2016;16:8.

19. Voloshin T, Davidi S, Porat Y, et al. Immunomodulatory effect of tumor treating fields (TTFields) results in enhanced antitumor efficacy when combined with antiPD-1 therapy in mouse model of lung cancer. Int J Radiat Oncol Biol Phys 2019;104:237.

20. Postow MA, Callahan MK, Barker CA, et al. Immunologic correlates of the abscopal effect in a patient with melanoma. N Engl J Med 2012;366:925-31.

21. Wong ET, Lok E, Gautam S, et al. Dexamethasone exerts profound immunologic interference on treatment efficacy for recurrent glioblastoma. Br J Cancer 2015;113:1642.

22. Optune Elevate Expectations. Inovitro ${ }^{\mathrm{TM}}$. TTFields Lab Bench System. Available online: http://www.ssshare.it/tiSq

23. Lok E, Swanson KD, Wong ET. Tumor treating fields therapy device for glioblastoma: physics and clinical practice considerations. Expert Rev Med Devices 2015;12:717-26.

24. Wenger C, Salvador R, Basser PJ, et al. Modeling tumor 
treating fields (TTFields) application within a realistic human head model. Annu Int Conf IEEE Eng Med Biol Soc 2015;2015:2555-8.

25. Lok E, San P, Hua V, et al. Analysis of physical characteristics of Tumor Treating Fields for human glioblastoma. Cancer Med 2017;6:1286-300.

26. Lok E, San P, White V, et al. RTHP-12. Comparative analysis of tumor treating fields using conventional versus alternative array placement for posterior fossa glioblastoma. Neuro Oncol 2018;20:vi227-8.

27. Wong ET, Hess KR, Gleason MJ, et al. Outcomes and prognostic factors in recurrent glioma patients enrolled onto phase II clinical trials. J Clin Oncol 1999;17:2572-8.

28. Stupp R, Wong ET, Kanner AA, et al. NovoTTF-100A versus physician's choice chemotherapy in recurrent glioblastoma: a randomised phase III trial of a novel treatment modality. Eur J Cancer 2012;48:2192-202.

29. Mrugala MM, Engelhard HH, Dinh Tran D, et al. Clinical practice experience with NovoTTF-100ATM system for glioblastoma: the Patient Registry Dataset (PRiDe). Semin Oncol 2014;41 Suppl 6:S4-13.

30. Kanner AA, Wong ET, Villano JL, et al. Post Hoc analyses of intention-to-treat population in phase III comparison of NovoTTF-100ATM system versus best physician's choice chemotherapy. Semin Oncol 2014;41 Suppl 6:S25-34.

31. Stupp R, Taillibert S, Kanner AA, et al. Maintenance therapy with tumor-treating fields plus temozolomide vs temozolomide alone for glioblastoma: a randomized clinical trial. JAMA 2015;314:2535-43.

32. Fayers P, Bottomley A, EORTC Quality of Life Group, et al. Quality of life research within the EORTC-the EORTC QLQ-C30. European Organisation for Research and Treatment of Cancer. Eur J Cancer 2002;38 Suppl 4:S125-33.

33. Taphoorn MJB, Dirven L, Kanner AA, et al. Influence of treatment with tumor-treating fields on healthrelated quality of life of patients with newly diagnosed glioblastoma: a secondary analysis of a randomized clinical trial. JAMA Oncol 2018;4:495-504.

34. Zhu JJ, Demireva P, Kanner AA, et al. Health-related quality of life, cognitive screening, and functional status in a randomized phase III trial (EF-14) of tumor treating fields with temozolomide compared to temozolomide alone in newly diagnosed glioblastoma. J Neurooncol 2017;135:545-52.

35. Toms SA, Kim CY, Nicholas G, Ram Z. Increased compliance with tumor treating fields therapy is prognostic for improved survival in the treatment of glioblastoma: a subgroup analysis of the EF-14 phase III trial. J
Neurooncol 2019;141:467-73.

36. Ballo MT, Urman N, Lavy-Shahaf G, et al. Correlation of Tumor Treating Fields Dosimetry to Survival Outcomes in Newly Diagnosed Glioblastoma: A Large-Scale Numerical Simulation-Based Analysis of Data from the Phase 3 EF-14 Randomized Trial. Int J Radiat Oncol Biol Phys 2019;104:1106-13.

37. Wong ET, Lok E, Swanson KD. Clinical benefit in recurrent glioblastoma from adjuvant NovoTTF-100A and TCCC after temozolomide and bevacizumab failure: a preliminary observation. Cancer Med 2015;4:383-91.

38. Lu G, Rao M, Zhu P, et al. Triple-drug therapy with bevacizumab, irinotecan, and temozolomide plus tumor treating fields for recurrent glioblastoma: a retrospective study. Front Neurol 2019;10:42.

39. U.S. Food and Drug Administration. Premarket Approval (PMA). Available online: https://www.accessdata.fda.gov/ scripts/cdrh/cfdocs/cfpma/pma.cfm?id=P100034S01

40. Stachelek GC, Grimm J, Moore J, et al. Abstract 676: Preliminary investigation into the dosimetric impact of tumor treating field arrays on concurrent radiotherapy for newly-diagnosed glioblastoma. Cancer Res 2018;78:Abstract nr 676.

41. Grossman R, Limon D, Bokstein F, et al. Abstract CT008: Tumor treating fields combined with radiotherapy and temzolomide for newly diagnosed glioblastoma: final results from a pilot study. Cancer Res 2019;79:Abstract nr CT008.

42. Wong ET, Swanson KD. Dexamethasone-friend or foe for patients with glioblastoma? JAMA Neurol 2019;76:247-8.

43. Tomić S, Joksimović B, Bekić M, et al. Prostaglanin-E2 Potentiates the Suppressive Functions of Human Mononuclear Myeloid-Derived Suppressor Cells and Increases Their Capacity to Expand IL-10-Producing Regulatory T Cell Subsets. Front Immunol 2019;10:475.

44. Cloughesy TF, Landolfi J, Hogan DJ, et al. Phase 1 trial of vocimagene amiretrorepvec and 5-fluorocytosine for recurrent high-grade glioma. Sci Transl Med 2016;8:341ra75.

45. Tocagen Inc. Tocagen Reports Results of Toca 5 Phase 3 Trial in Recurrent Brain Cancer. Available online: https:// www.prnewswire.com/news-releases/tocagen-reportsresults-of-toca-5-phase-3-trial-in-recurrent-braincancer-300916705.html

Cite this article as: Berger TR, Wong ET. Tumor treating fields in neuro-oncology: integration of alternating electric fields therapy into promising treatment strategies. Chin Clin Oncol 2021;10(4):36. doi: 10.21037/cco.2020.02.04 